\title{
Preface to the Special Issue on "High Nitrogen Steels"
}

\author{
Makoto KIKUCHI and Yoshinao MISHIMA ${ }^{1)}$ \\ Chairman of the Organizing Committee of The Fourth International Conference on High Nitrogen Steels; Tokyo Institute of \\ Technology, Ookayama, Meguro-ku, Tokyo, 152 Japan. \\ 1) Editor of ISIJ International; Tokyo Institute of Technology, Nagatsuta, Midori-ku, Yokohama, Kanagawa-ken, 226 Japan.
}

This issue of ISIJ International contains 37 papers presented at "The Fourth International Conference on High Nitrogen Steels 〈HNS '95)" held in Kyoto, Japan, from September 26 to $28,1995$.

Effects of nitrogen in steels, both detrimental and beneficial, have long been a subject of studies. Y. Imai, Professor Emeritus of Tohoku University, is one of the prominent researchers in this field, who made a series of studies on the effects of nitrogen on physical, chemical and mechanical properties of steels between the forties and sixties. Some beneficial effects led to the studies of highly alloyed nitrogen steels that cannot be produced by ordinary melting under ambient pressure.

M. Okamoto, the late Professor of Tokyo Institute of Technology, made pioneering studies on high nitrogen stainless steels produced by high pressure melting in the fifties and sixties. Since then, Japanese interest in high nitrogen steels has been dwindling. This reflects the marginal Japanese contribution in this conference, which is quite unusual in the conference on steels held in Japan. Unlimited demands for advanced steels for special use renewed the interest in high nitrogen steels in Europe in the eighties, which led to the first international conference on high nitrogen steels in Lille, France in May 1988, organized by J. Foct and A. Hendry. This Kyoto conference followed the third one held in Kiev, Ukraine in September 1993. It was the first one held in other place than Europe and was sponsored by The Iron and Steel Institute of Japan.

This conference attracted a reasonable size of the participants from all over the world and many high quality papers were presented by leading authorities in this field. One hundred thirty attendants from eighteen countries participated in the conference. Fifty-eight oral papers including eight keynote lectures were presented in the eleven sessions including the evening session on the first day. Thirty-seven poster papers were presented in a poster session, to which all afternoon on the second day was exclusively devoted. Most of the papers represent the-present-state-of-the-art in high nitrogen steels.

The organizers wish to express gratitude to all the speakers, contributing authors and many other participants who made this conference a success. Deepest gratitude is due to those who gave Keynote Lectures: H. Borne, Ruhr University Bochum; V. G. Gavriljuk, Institute of Metal Physics Ukraine; H. J. Grabke, Max-Plank Institute for Iron and Steel Research; T. Nakazawa, Nippon Steel Corp.; K. Osozawa, Nippon Yakin Kogyo Co.; M. O. Speidel, Swiss Federal Institute of Technology Zurich and G. Stein, VSG Energie- und Schmiedetechnik GmbH. The organizers also wish to thank the members of the Scientific Committee and the chairmen of the sessions for their efforts. Special thanks are due to T. Ando, The Iron and Steel Institute of Japan, for his secretarial work, to Y. Kimura, K. Araki, T. Kashiwa and R. Yokote, Tokyo Institute of Technology, for their work to run the conference smoothly during the meeting, and to T. Matsuo and M. Takeyama, Tokyo Institute of Technology, for their untiring efforts during all phases of holding the conference.

The organizers wish to acknowledge the financial support from Grant-in-Aid for Publication of Scientific Research Results, Grant-in-Aid for Scientific Research, The Ministry of Education, Science, Sports and Culture.

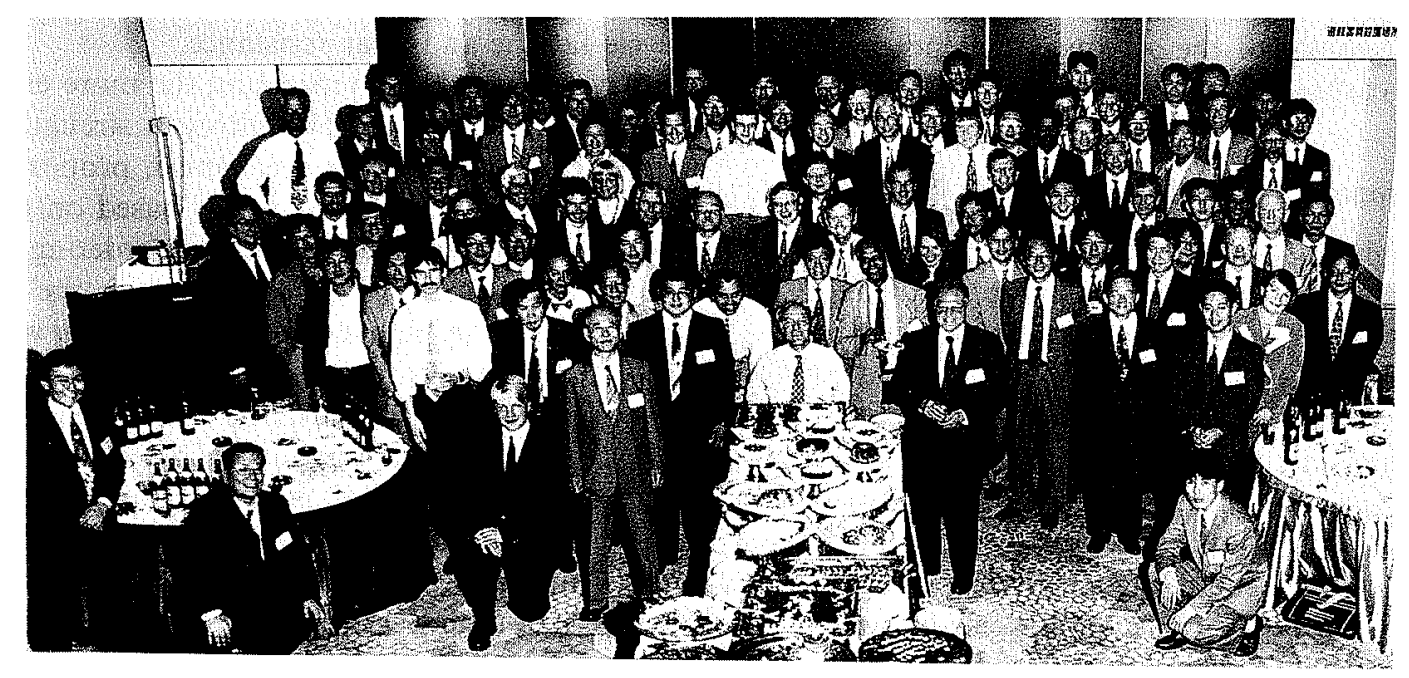

Participants to the 4th International Conference on High Nitrogen Steels at Farewell Party 